\title{
Spin-Dependent Phenomena in Magnetoelectronic Devices
}

\author{
J. BARnAŚ ${ }^{a, b, *}$, M. GMitra ${ }^{c}$, W. RUdZIŃSKI ${ }^{a}$, \\ M. WAWrzyniak ${ }^{a}$, V.K. Dugaev ${ }^{d}$ And H. Kunert ${ }^{e}$ \\ ${ }^{a}$ Department of Physics, Adam Mickiewicz University \\ Umultowska 85, 61-614 Poznań, Poland \\ ${ }^{b}$ Institute of Molecular Physics, Polish Academy of Sciences \\ Smoluchowskiego 17, 60-179 Poznań, Poland \\ ${ }^{c}$ Department of Theoretical Physics and Astrophysics, P.J. Šafárik University \\ Park Angelinum 9, 04001 Košice, Slovak Republic \\ ${ }^{d}$ Department of Physics and Applied Physics, Rzeszów University of Technology \\ Al. Powstańców Warszawy 6, 35-959 Rzeszów, Poland \\ ${ }^{e}$ Department of Physics, University of Pretoria \\ 0002 Pretoria, Republic of South Africa

\begin{abstract}
Spin effects in electronic transport properties of artificial magnetic structures, like nanopillar spin valves, tunnel junctions, mesoscopic double-barrier junctions (single-electron transistors) are briefly discussed. Two classes of spin effects are distinguished; i.e. magnetoresistance phenomena due to magnetization rotation, and current-induced magnetic switching and magnetic dynamics.
\end{abstract} \\ PACS numbers: 75.60.Ch, 75.70.Cn, 75.70.Pa
}

\section{Introduction}

The existence of two non-equivalent and well-defined spin channels for electronic transport is an intrinsic property of $3 d$ ferromagnetic metals. This nonequivalence has a significant influence on transport characteristics in spatially nonuniform magnetic systems. The associated phenomena can be classified into two categories. The first class includes the magnetoresistance phenomena, i.e. the dependence of charge current flowing through a system on its magnetic configuration [1-3]. The second category includes the phenomena which describe the influence of charge current flowing through a system on its magnetic state [4].

${ }^{*}$ corresponding author 
All these phenomena have been observed in magnetic metallic multilayers [5, 6]. However, similar phenomena also can be observed in other kinds of artificial magnetic systems, for instance in planar single-barrier tunnel junctions, planar doublebarrier tunnel junctions, mesoscopic double-barrier junctions in the form of a single-electron transistor, etc. In the latter case the central electrode (sometimes called also an island) can be in the form of a metallic nanoparticle, semiconductor quantum dot, molecule, etc. In the following we will briefly discuss both classes of the transport phenomena in magnetic nanopillars and in double-barrier tunnel junctions.

\section{Magnetic nanopillars}

\subsection{Influence of magnetic state on current}

A magnetic nanopillar consists of two small-area magnetic layers separated by a nonmagnetic metallic spacer layer. The system is contacted on both sides by nonmagnetic metallic leads. We assume that magnetic moment of the left (reference) layer is fixed along the corresponding in-plane easy axis, while magnetic moment of the right (sensing) layer is free to rotate and its orientation is described by the polar $\theta$ and azimuthal $\varphi$ angles. The local quantization axes in the ferromagnetic films are along the local spin moments. Apart from this, the charge current $I$ is positive by definition when it flows from left to right, i.e., from the reference layer towards the sensing one.

Electrical resistance of such a spin valve depends on relative orientation of the magnetic moments, i.e. on the angles $\theta$ and $\varphi$. Experimentally one usually measures variation of the resistance when magnetic configuration varies from antiparallel $\left(R_{\mathrm{ap}}\right)$ to parallel $\left(R_{\mathrm{p}}\right)$ one. The relative magnetoresistance is then described by the ratio $\left(R_{\mathrm{ap}}-R_{\mathrm{p}}\right) / R_{\mathrm{p}}$, and is known as the giant magnetoresistance (GMR) effect [1-3]). The effect is a consequence of the spin asymmetry of the two spin channels, and this asymmetry is quantified by two parameters - the bulk spin asymmetry factor $\beta$ and the interface spin asymmetry parameter $\gamma$.

The effective spin asymmetry of a particular magnetic layer is a superposition of both bulk and interface spin asymmetries. When the effective spin asymmetries of both magnetic films have the same sign, the resistance of a nanopillar is larger in the antiparallel configuration and smaller in the parallel one (this is so-called normal GMR effect). When, however, the spin asymmetries of both layers have opposite signs, the resistance is larger in the parallel configuration and smaller in the antiparallel one (inverse GMR effect). Both, normal and inverse GMR effects have been observed experimentally [7].

Generally, one can define GMR for an arbitrary noncollinear configuration as the relative difference of the resistances in the antiparallel and noncollinear configurations. Usually one measures GMR for in-plane magnetization. In symmetrical structures, the GMR varies then with the angle $\theta$ between magnetic moments as $\sin ^{2} \theta / 2$. In a general case, for instance in asymmetrical systems, the angular variation becomes more complex, and GMR can vanish and change sign at 
a certain non-collinear configuration. This angular variation is also sensitive to the conduction mechanism and may be different in ballistic and diffusive transport regimes [8]. Moreover, GMR also depends on the orientation of the charge current, and is usually larger for current flowing normally to the plane of the layers (CPP geometry) than in the case of in-plane current (CIP geometry).

The mechanism of GMR in the CPP geometry includes one important ingredient which is absent in the CIP geometry, namely spin accumulation (spin splitting of the electrochemical potentials) at the interfaces, which extends to the distance of the order of spin diffusion length from the interface. Spatial variation of the spin accumulation in ferromagnetic layers obeys the diffusion equation [9],

$$
\frac{\partial^{2}\left(\bar{\mu}_{\uparrow}-\bar{\mu}_{\downarrow}\right)}{\partial x^{2}}=\frac{1}{l_{\mathrm{sf}}^{2}}\left(\bar{\mu}_{\uparrow}-\bar{\mu}_{\downarrow}\right),
$$

where $\bar{\mu}_{\uparrow}$ and $\bar{\mu}_{\downarrow}$ are the electro-chemical potentials for spin-majority and spin-minority electrons, and $l_{\mathrm{sf}}$ is the spin diffusion length. The electro-chemical potentials can be written in the form $\check{\bar{\mu}}=\bar{\mu}_{0} \check{1}+g \check{\sigma}_{z}$, with $\bar{\mu}_{0}=\frac{1}{2}\left(\bar{\mu}_{\uparrow}+\bar{\mu}_{\downarrow}\right)$, and $g=\frac{1}{2}\left(\bar{\mu}_{\uparrow}-\bar{\mu}_{\downarrow}\right)$. The quantity $g$ describes spin splitting of the electrochemical potential, i.e. spin accumulation. The current matrix $\check{j}$ in spin space can be written as $\breve{j}=\frac{1}{2}\left(j_{0} \check{1}+j_{z} \check{\sigma}_{z}\right)$, where $j_{0}=\left(j_{\uparrow}+j_{\downarrow}\right)$ is the total particle current density, and $j_{z}=\left(j_{\uparrow}-j_{\downarrow}\right)$ is the $z$-component of the spin current density. The spin accumulation and spin current have only $z$-components in magnetic films (quantization axis, i.e. the $z$-axis is along the local spin polarization). In nonmagnetic films all components of the spin accumulation and spin current in the local reference frames of the left and right magnetic films are nonzero in noncollinear configurations.

\subsection{Influence of current on magnetic state}

When orientation of the local magnetic moments varies in space, a nonzero net spin (angular) momentum can be locally transferred from conduction electrons to local magnetic moments. This appears as a torque exerted locally on the magnetization. In spin valve structures the spin-transfer torque may lead to the phenomenon of current-induced magnetic switching (CIMS), predicted first theoretically [4], and then confirmed experimentally [5]. The magnetic switching can be detected by measuring resistance change of the system. The switching between low and high resistive states has been observed for currents exceeding certain critical values and for sufficiently low external fields. For larger fields, transition to steady precessional regime has been found [6].

When magnetic moments of the two layers form a canted configuration, spin orientation of an electron leaving a particular layer is not adjusted to the spin quantization axis of the second layer and becomes fitted to this quantization axis on the distance of the order of the Fermi wavelength (a few atomic planes in the case of metallic layers) from the interface [10]. From the above it follows that the perpendicular component of the spin current entering a particular magnetic layer is totally or partly absorbed by the layer. The absorbed spin is equivalent to the torque $\boldsymbol{\tau}$ exerted on the ferromagnetic film. This torque (per unit square) can be 
calculated as

$$
\tau=\frac{\hbar}{2}\left(j_{\perp \mathrm{L}}-j_{\perp R}\right)
$$

where $j_{\perp \mathrm{L}}$ and $j_{\perp \mathrm{R}}$ are the normal (to the magnetization) components of the spin current density at the left and right interfaces of the magnetic film, calculated on the normal metal side of these interfaces.

The in-plane component $\boldsymbol{\tau}_{\theta}$ of the torque acting on the sensing magnetic layer can be written as $\boldsymbol{\tau}_{\theta}=a I \hat{\boldsymbol{s}} \times(\hat{\boldsymbol{s}} \times \hat{\boldsymbol{S}})$, where $\hat{\boldsymbol{s}}$ and $\hat{\boldsymbol{S}}$ are the unit vectors along the spin polarization of the sensing and reference magnetic layers, respectively, and $a$ is a parameter. The torque $\tau_{\theta}$ is defined in such a way that positive torque tends to increase the angle $\theta$, whereas negative torque tends to decrease $\theta$. In turn, the out-of-plane component $\boldsymbol{\tau}_{\varphi}$ of the torque may be written as $\boldsymbol{\tau}_{\varphi}=b I \hat{\boldsymbol{s}} \times \hat{\boldsymbol{S}}$, where $b$ is the relevant parameter.

Exemplary numerical results on the in-plane and out-of-plane components of the torque in the diffuse transport regime, as well as on the corresponding parameters $a$ and $b$, are shown in Fig. 1a,b, where the torque is normalized to current. Since the spin accumulation and spin currents are linear in the charge current, the curves in Fig. 1a are the same for an arbitrary magnitude of the charge current $I$. Let us note that the sign of torque changes when $I$ is reversed. Figure 1 implies that a positive current $(I>0)$ tends to destabilize the parallel configuration and can switch it to antiparallel one when current exceeds a certain threshold value. On the other hand a negative current tends to destabilize the antiparallel configuration and stabilize the parallel one [5, 7].
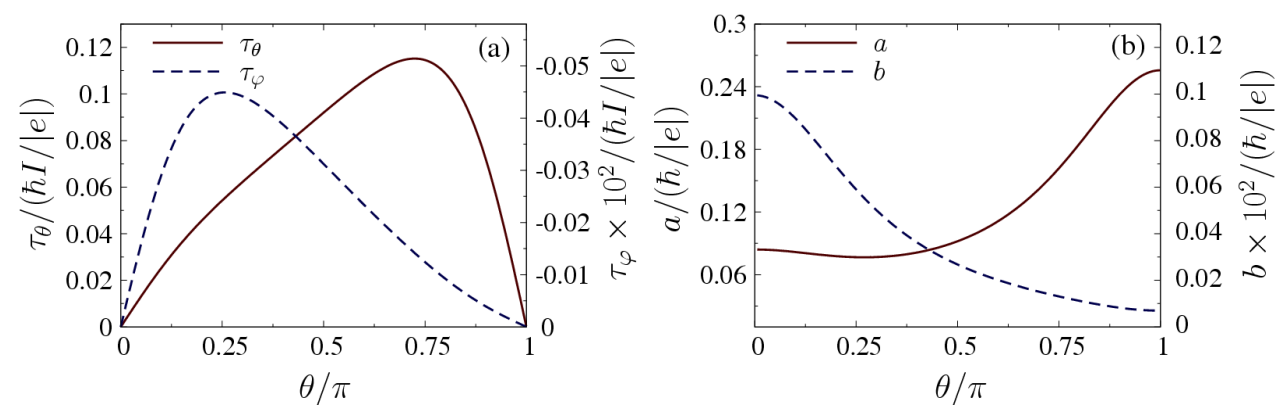

Fig. 1. Spin-transfer characteristics for the $\mathrm{Cu} / \mathrm{Co}(20) / \mathrm{Cu}(10) / \mathrm{Co}(8) / \mathrm{Cu}$ spin valve (numbers in parentheses denote layer thickness in $\mathrm{nm}$ ): (a) normalized torques $\tau_{\theta}$ and $\tau_{\varphi}$ acting on the sensing layer, $\operatorname{Co}(8)$, calculated as a function of the angle $\theta$ for the parameters typical of the $\mathrm{Co} / \mathrm{Cu}$ system; (b) the corresponding angular dependence of the parameters $a$ and $b$.

When positive current (flowing from the reference towards the sensing layer) causes transition to antiparallel magnetic configuration, the switching is known as normal CIMS. In turn, when positive current induces transition to parallel config- 
uration, it is called inverse CIMS. Reversing bias polarization one can also reverse the magnetic switching. It has been shown that the spin asymmetry factor of the reference layer determines whether the switching is normal or inverse; when this factor is positive, the switching is normal, and when it is negative, the switching is inverse [11].

In standard spin valves (those which show either normal or inverse currentinduced switching in the absence of external magnetic field), transition to stationary precessional states is possible only in a sufficiently strong external magnetic field. When current is above the critical value and tends to rotate magnetic moment of the sensing layer, while the applied magnetic fields tends to restore its original orientation, competition of these two torques leads to a stationary precessional state. Although the damping term (Gilbert damping) leads to energy dissipation, the energy is continuously pumped from the voltage source to the magnetic system and supports microwave oscillations.

To describe dynamical behavior of the sensing layer one can use the LandauLifshitz-Gilbert equation, which additionally includes the torque due to spin-transfer. The fundamental frequency (first harmonics) of the current-induced oscillations, calculated within the macro spin model, first decreases with increasing current (red shift), and then increases (blue shift) revealing a profound minimum. This minimum is associated with bifurcation of the large-angle in-plane (LIP) precessions into two so-called out-of-plane (OP) orbits.

In symmetric systems discussed above the current-induced precessional states appear in relatively large external magnetic fields. In asymmetric structures which exhibit non-standard angular dependence of the in-plane spin torque, like $\mathrm{Co} / \mathrm{Cu} / \mathrm{Py}$ systems, the transition to precessional states can occur also in zero magnetic field [12]. Such systems are of particular interest as they may be used as microwave nanogenerators entirely controlled by electric field.

\section{Tunnel junctions}

An effect similar to the GMR also occurs when the nonmagnetic metallic spacer layer is replaced with a nonmagnetic insulating barrier. This effect was discovered a long time ago in ferromagnetic planar junctions by Jullière [13], and is of current interest due to applications in magnetic storage technology (magnetic random access memories). As in the case of GMR, the tunnel magnetoresistance (TMR) consists in a decrease (increase is also possible) in the junction resistance when magnetic configuration of the junction varies from antiparallel to parallel. CIMS in single planar tunnel junctions has also been observed [14].

Tunneling in more complex junctions, particularly in mesoscopic ones, where charging effects become important, is of particular interest due to the interplay of charge and spin degrees of freedom $[15,16]$. A specific kind of such systems are double-barrier junctions with a small central electrode (called an island). Such systems are known as single-electron transistors (SETs), mainly because electrons 
in a biased device flow one by one and the transfer of single electrons can be controlled by a gate voltage. In the case of sufficiently large metallic islands (but still in the nanometer range), a discrete structure of the electronic states in the grain is irrelevant. To observe the discrete electronic states in transport characteristics one should either diminish size of the metallic nanoparticles [17], or use semiconducting quantum dots based on two-dimensional electron gas [18]. Another group of single-electron devices are molecular ferromagnetic transistors, and especially ferromagnetically contacted carbon nanotubes [19].

Variation of electrical resistance (conductance) with magnetic configuration of double-barrier tunnel junctions (SETs) has been investigated experimentally and theoretically. The main advantage of such systems is that conductance and TMR can be controlled by a gate voltage, which makes them very promising for future applications. Some exemplary numerical results on TMR in the linear response regime (zero bias limit) are shown in Fig. 2 for a SET based on a singlelevel quantum dot attached to ferromagnetic leads. This figure clearly shows that TMR is strongly sensitive to the position of a discrete energy level (which may be controlled by a gate voltage) of the central part. When the levels $\epsilon_{d}$ and $\epsilon_{\mathrm{d}}+U$ are well below or above the Fermi level, TMR tends to the Jullière value [13], otherwise it is significantly reduced.

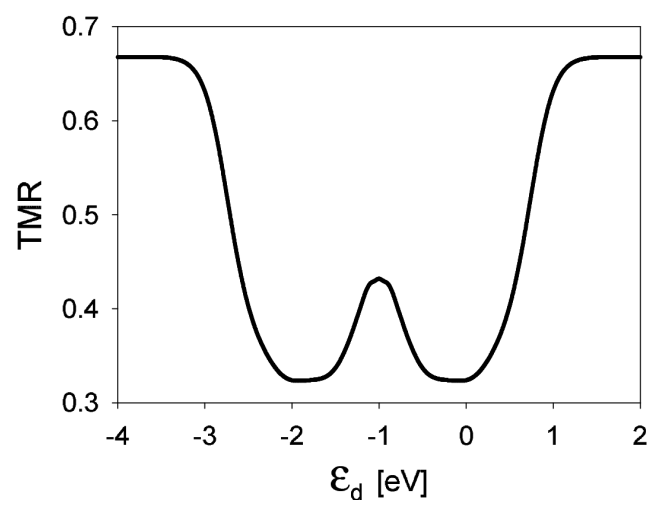

Fig. 2. TMR in the linear response regime,calculated as a function of the energy level $\epsilon_{d}$ of a single-level quantum dot in the Hartree-Fock approximation. The parameters assumed are: the Coulomb parameter $U=2 \mathrm{eV}$ (describing electrostatic energy of two electrons with opposite spins residing in the dot level), temperature $T=100 \mathrm{~K}$, leads' spin polarization factor $p=0.5$, and parameter $\Gamma=0.01 \mathrm{eV}$ (describing coupling of the dot to ferromagnetic leads).

As magnetoresistance phenomena in SETs have been extensively considered theoretically and also experimentally, much less has been done on a current-induced switching in such double-barrier systems. Anyway, theoretical considerations show that such a switching is possible when the island is either a magnetic particle or a magnetic molecule [20]. 


\section{Acknowledgments}

This work was supported by funds from the Ministry of Science and Higher Education as research projects in the years 2006-2009 and 2006-2008, and by Slovak Ministry of Education as a research project MVTS POL/SR/UPJS07.

\section{References}

[1] M.N. Baibich, J.M. Broto, A. Fert, F.N. Van Dau, F. Petroff, P. Eitenne, G. Creuzet, A. Friederich, J. Chazelas, Phys. Rev. Lett. 61, 2472 (1988).

[2] G. Binasch, P. Grünberg, F. Saurenbach, W. Zinn, Phys. Rev B 39, 4828 (1989).

[3] J. Barnaś, A. Fuss, R.E. Camley, P. Grünberg, W. Zinn, Phys. Rev. B 42, 8110 (1990).

[4] J.C. Slonczewski, J. Magn. Magn. Mater. 159, L1 (1996); 195, L261 (1999); L. Berger, Phys. Rev. B 54, 9353 (1996).

[5] J.A. Katine, F.J. Albert, R.A. Buhrman, E.B. Myers, D.C. Ralph, Phys. Rev. Lett. 84, 3149 (2000).

[6] S.I. Kiselev, J.C. Sankey, I.N. Krivorotov, N.C. Emley, R.J. Schoelkopf, R.A. Buhrman, D.C. Ralph, Nature (London) 425, 380 (2003).

[7] M. AlHajDarwish, H. Kurt, S. Urazhdin, A. Fert, R. Loloee, W.P. Pratt, Jr., Phys. Rev. Lett. 93, 157203 (2004).

[8] J. Barnaś, O. Baksalary, A. Fert, Phys. Rev. B 56, 6079 (1997).

[9] T. Valet, A. Fert, Phys. Rev. B 48, 7099 (1993).

[10] M.D. Stiles, A. Zangwill, Phys. Rev. B 66, 014407 (2002); J. Appl. Phys. 91, 6812 (2002).

[11] J. Barnaś, A. Fert, M. Gmitra, I. Weymann, V.K. Dugaev, Phys. Rev. B 72, 024426 (2005).

[12] M. Gmitra, J. Barnaś, Phys. Rev. Lett. 96, 207205 (2006).

[13] M. Jullière, Phys. Lett. A 54, 225 (1975).

[14] J. Hayakawa, S. Ikeda, Y.M. Lee, R. Sasaki, T. Meguro, F. Matsukura, H. Takahashi, H. Ohno, Jpn. J. Appl. Phys. 45, L1057 (2006).

[15] J. Barnaś, A. Fert, Phys. Rev. Lett. 80, 1058 (1998).

[16] K. Ono, H. Shimada, S. Kobayashi, Y. Outuka, J. Phys. Soc. Jpn. 65, 3449 (1996).

[17] M.M. Deshmukh, D.C. Ralph, Phys. Rev. Lett. 89, 266803 (2002).

[18] K. Hamaya, S. Masubuchi, M. Kawamura, T. Machida, M. Jung, K. Shibata, K. Hirakawa, T. Taniyama, S. Ishida, Y. Arakawa, Appl. Phys. Lett. 91, 22107 (2007).

[19] K. Tsukagoshi, B.W. Alphenaar, H. Ago, Nature 401, 572 (1999).

[20] M. Misiorny, J. Barnaś, Phys. Rev. B 75, 134425 (2007); 76, 54448 (2007). 